\title{
Enhancing MAC performance of DCF protocol for IEEE 802.11 wireless LANs
}

\author{
Woo-Yong Choi
}

\begin{abstract}
The DCF (Distributed Coordination Function) is the basic MAC (Medium Access Control) protocol of IEEE 802.11 wireless LANs and compatible with various IEEE 802.11 PHY extensions. The performance of the DCF degrades exponentially as the number of nodes participating in the DCF transmission procedure increases. To deal with this problem, we propose a simple, however efficient modification of the DCF by which the performance of the DCF is greatly enhanced.

K e y w or d s: wireless LAN, MAC, DCF, throughput
\end{abstract}

\section{Introduction}

When nodes contend to transmit their data frames using the DCF (Distributed Coordination Function) in IEEE 802.11 wireless LANs, assuming that only backoff stage exists and the length and transmission rate of data frames are fixed, from the formulas (1) to (7) in [1] the MAC (Medium Access Control) throughput can be approximated by

$$
\frac{n s(1-w)^{n-1} L}{(1-w)^{n}+\left(1-(1-w)^{n}\right) T}, \quad \text { with: } w=\frac{2}{W_{i}+1}
$$

where $W_{i}+1$ is the maximum backoff time slots of backoff stage $i, L$ the length of payloads in data frames and $T$ the time taken to transmit a data frame and the corresponding ACK frame including the inter-frame spacings of DIFS (DCF Inter-Frame Space) and SIFS (Short Inter-Frame Space). It can be seen from (1) that the performance of backoff stage $i$ degrades exponentially as the number of nodes participating in the DCF transmission procedure increases. Therefore, we deduce that the performance of the DCF even with backoff stages $0,1 \ldots, m$ degrades exponentially as the number of nodes increases. With the increased number of users of wireless LANs by the new technologies such as IEEE 802.11n and IEEE 802.11ac, and the deployment of WiFi sensors, the research is needed to enhance the MAC performance of wireless LANs [2-5].

In the literature, the optimization of contention window and the number of backoff stages has been studied to enhance the performance of the DCF [6-8]. However, because the boundary of candidates for the optimization of the parameters converges to infinite limit as the number of nodes participating in the DCF transmission procedure increases, it is difficult to develop an efficient method for optimizing the parameters. According to [6], the joint optimization of the parameters of contention window and the number of backoff stages yields no additional performance gain over the simple optimization of contention window.

In this paper, we want to let with the probability $p$ each node in backoff stage 0 be able to attempt to transmit its data frame after a PIFS (PCF Inter-Frame Space) period following the detection of idle channel state without backoff. Because a PIFS period is smaller than a DIFS period which is the minimum waiting time for general nodes before attempting to transmit their data frames, the nodes in backoff stage 0 that are selected with the probability $p$ are privileged for the prioritized channel access. By the prioritized channel ac-cess of backoff stage 0 , the transitions of nodes from other backoff stages than backoff stage 0 to backoff stage 0 are reduced and the transitions from backoff stage 0 to other backoff stages are increased, which leads to the reduced number of nodes in backoff stage 0 . The proposed method differentiates the nodes in backoff stage 0 and other backoff stages into different contention domains and has the advantage of reducing the collisions between transmissions by granting the prioritized channel access to the nodes in backoff stage 0 . We want to maximize the MAC performance of wireless LANs by optimizing the probability $p$ that the nodes in backoff stage 0 are granted the prioritized channel access considering the MAC throughput and the service fairness between the nodes in backoff stage 0 and other backoff stages.

We propose the new method for enhancing the MAC performance of the DCF in IEEE 802.11 wireless LANs. Simulation results are presented to show the MAC performance improvement by the proposed method.

\footnotetext{
* Department of Industrial and Management Systems Engineering, Dong-A University, Nakdong-daero, Saha-gu, Busan, Korea, wychoi77@dau.ac.kr
} 


\section{MAC performance improvement method}

The authentication and association processes should be performed between the AP (Access Point) and the nodes before the nodes are allowed to be served in IEEE 802.11 wireless LANs. Therefore, the AP knows the number $n$ of nodes that are associated with itself. The AP broadcasts the beacon frames regularly during CPs (Contention Periods) where the DCF is operated. For the proposed method, the new field of the updated prob-ability that the nodes in backoff stage 0 are granted the prioritized channel access should be added to the existing beacon frames so that the nodes update the probability $p$ from the received beacon frames.

Let the nodes in backoff stage 0 generate the random numbers in $[0,1]$. The nodes that generated the random numbers $\leq p$ are privileged to attempt to transmit their data frames after a PIFS period following the detection of idle channel state. Because general nodes should wait for a DIFS period, which is greater than a PIFS period by a time slot, before attempting to transmit their data frames, the nodes in backoff stage 0 that are selected with the probability $p$ are granted the prioritized channel access. When the nodes granted the prioritized channel access experience successful data transmissions or experience unsuccessful data transmissions due to the collisions of simultaneous transmissions or any other reasons, the nodes follow the existing DCF backoff pro-cedure for the transition between backoff stages after successful or unsuccessful data transmissions. The nodes in backoff stage 0 that are not selected for the prioritized channel access and the nodes in backoff stages other than 0 follow the existing DCF backoff procedure to attempt to transmit their data frames. The AP can optionally access the channel after a SIFS period following the detection of idle channel state. Because a SIFS period is smaller than a PIFS period by a time slot, the AP can safely broadcast the beacon frames from which the nodes can update the probability $p$.

Let us assume that $r$ nodes out of $n$ nodes are in backoff stage 0 and the remaining $n-r$ nodes are in other backoff stages. Because $r$ nodes in backoff stage 0 attempt to transmit their data frames using the prioritized channel access with probability $p$, the probability $q$ that the transmission using the prioritized channel access is successful is upper bounded as follows

$$
q \leq r p(1-p)^{r-1}
$$

where the inequality holds if the hidden node problem is present in wireless LANs or an error-prone channel is considered. As more consecutive successful transmissions occur using the prioritized channel access, the service fairness between the nodes in backoff stage 0 and other backoff stages is worse. The mean sum $s$ of the waiting times that the nodes in backoff stages other than 0 experience due to the consecutive successful transmissions using the prioritized channel access can be derived as follows

$$
s=\left(\frac{1}{1-q}-1\right) \leq\left(\frac{1}{1-r p(1-p)^{r-1}}-1\right)
$$

where $\frac{1}{1-q}-1$ is the mean number of consecutive successful transmissions using the prioritized channel access and $E\left[T_{s}\right]$ is the mean time taken to transmit a data frame and the corresponding ACK frame including the interframe spacings of PIFS and SIFS. The AP can derive by collecting the transmissions times of the data frames that it receives successfully from the nodes. Because the upper bound of the successful transmission probability $q$ in (2) is maximized when $p=\frac{1}{r}$, considering that $r=1,2, \ldots, n$ nodes are in backoff stage $0, p$ maximizing the upper bound of $q$ is ranged as follows

$$
p \in\left[\frac{1}{n}, 1\right]
$$

Furthermore, if we want the mean sum $s$ of the waiting times of the nodes in backoff stages other than 0 due to the prioritized channel access in (3) to be upper bounded by $D$, the probability $p$ should satisfy the following conditions

When $r=2,3, \ldots, n$, the upper bound of $p$ that satisfies (5) is 1 . When $r=1$, the upper bound of $p$ that satisfies (5) is

$$
p \leq 1-\frac{(n-1) E\left[T_{s}\right]}{(n-1) E\left[T_{s}\right]+D}
$$

Therefore, combining (4) and (6), when $n \geq 2, p$ is ranged as

$$
p \in\left[\frac{1}{n}, 1-\frac{(n-1) E\left[T_{s}\right]}{(n-1) E\left[T_{s}\right]+D}\right]
$$

When $n=1$, we can set $p$ to 1 . For convenience of explanation, the lower and upper bounds in (7) are, respectively, denoted by $p_{L}$ and $p_{U}$.

We need to select the prioritized channel access probability $p$ in the range of (7) so that the MAC throughput is maximized. For this purpose, for the current prioritized channel access probability $p$, a small constant $\alpha, p_{L}$ and $p_{U}$, the AP regularly measures the MAC throughputs of the wireless LAN while the modified DCF is operated with the changed prioritized channel access probabilities of

$$
\begin{aligned}
& p_{1}=\min \left(\max \left(p-\alpha, p_{L}\right), p_{U}\right) \\
& p_{2}=\max \left(\min \left(p+\alpha, p_{U}\right), p_{L}\right)
\end{aligned}
$$

each for amount of time $Y$. Then, $p$ is updated to be $p_{1}$ or $p_{2}$ so that the MAC throughput increases with the updated prioritized channel access probability. Initially, the AP measures the MAC throughput while the modifies DCF is operated with the prioritized channel access 


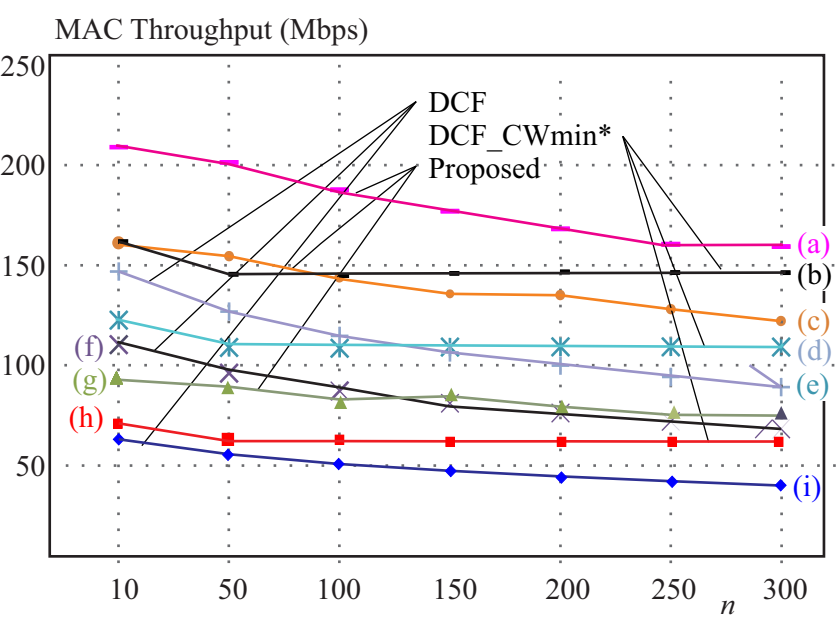

Fig. 1. MAC throughput results

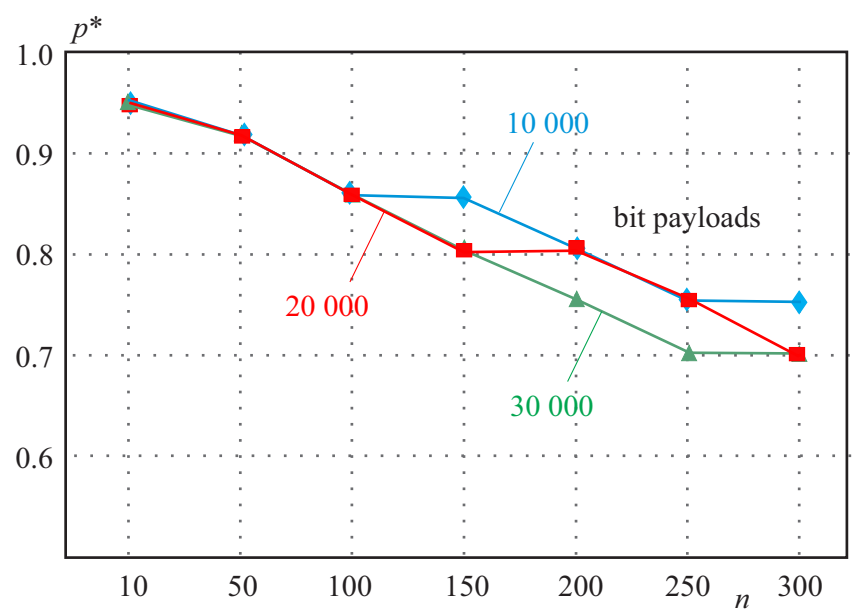

Fig. 3. Optimal prioritized channel access probability

probability of $p=p_{L}$ for amount of time $X$, and experiments with the prioritized channel access probabilities of $p_{1}$ and $p_{2}$ to update the prioritized channel access probability. Whenever the prioritized channel access probability is updated, the AP newly measures the MAC throughput while the modified DCF is operated with the updated prioritized channel access probability for amount of time $X$, and experiments with the prioritized channel access probabilities of $p_{1}$ and $p_{2}$ to continuously update the prioritized channel access probability.

\section{Simulation results}

Let us assume that in an IEEE 802.11n wireless LAN $n=10,50,100,150,200,250,300$ nodes exist and each node continuously attempts to transmit their data frames with payloads of 10000 bits, 20000 bits, or 30000 bits using the original DCF, the DCF with optimal CW min, or our proposed method in Section 2. Optimal CW min that maximizes MAC throughput was obtained by the exhaustive search method. It is assumed that the hidden node problem does not exist, therefore, the RTS (Request

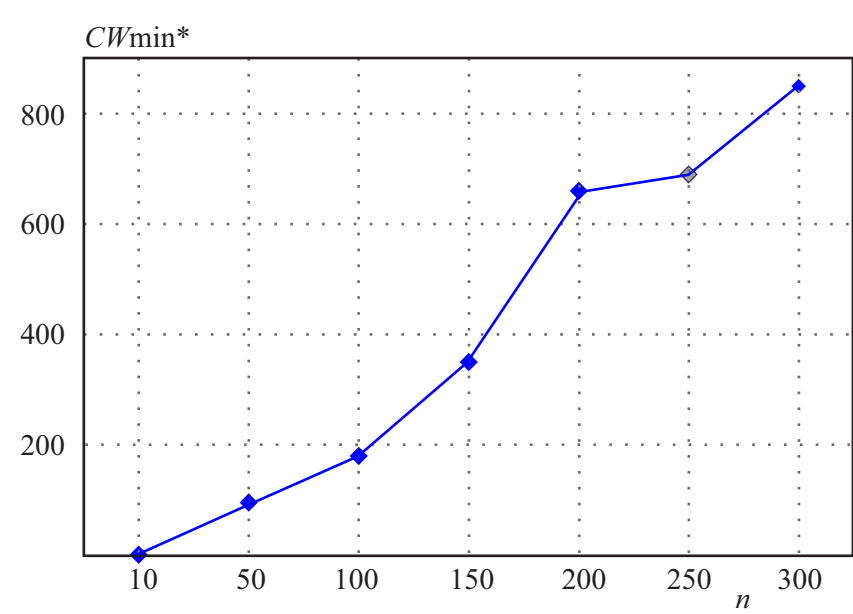

Fig. 2. Optimal CW min values

to Send) and CTS (Clear to Send) frames are not transmitted. The frame aggregation method is not employed and the transmission errors are assumed to occur only due to the collisions between transmissions. The values of $X$, $Y, \alpha$ and $D$ that is necessary for our proposed method and the values of other parameters used for simulations are presented in Table 1 [2].

Table 1. Values of simulation parameters

\begin{tabular}{cc}
\hline Parameters & Values \\
\hline Data transf. rate & $600 \mathrm{Mbps}$ \\
ACK transf. rate & $240 \mathrm{Mbps}$ \\
SIFS & $16 \mu \mathrm{s}$ \\
PIFS & $25 \mu \mathrm{s}$ \\
DIFS & $34 \mu \mathrm{s}$ \\
Time slot & $9 \mu \mathrm{s}$ \\
Max. Backoff Stage & 7 \\
PHY Header & $20 \mu \mathrm{s}$ \\
Trans. Lenghth & \\
$X$ & $900 \mathrm{~ms}$ \\
$Y$ & $100 \mathrm{~ms}$ \\
$\alpha$ & 0.05 \\
$D$ & $100 \mathrm{~ms}$ \\
\hline
\end{tabular}

In Fig. 1, we show the results of MAC throughputs of the original DCF, the DCF with optimal CW min and our proposed method for the combined cases of each $n$ and payload length. For obtaining each result of MAC throughput, at least 3000 data frames were generated in the transmission buffer of each node and simulations were conducted until the data frames in each transmission buffer were successfully transmitted. Especially for our proposed method, simulations were conducted until the prioritized channel access probability $p$ does not change and MAC throughput does not change more than $0.1 \%$. For this purpose, at least 300000 data frames were successfully transmitted for each case of simulation of our proposed method. The optimal CW min values used for 
the MAC throughput results of the DCF with optimal CW min are presented in Fig. 2, and the optimal prioritized channel access probability $p *$ values of our proposed method in Fig. 3. Optimal CW min increases as $n$ increases, however, does not change for each payload length, and $p *$ decreases as $n$ increases.

As can be seen in Fig. 1, as $n$ increases, the MAC throughputs of the original DCF, the DCF with optimal CW min and our proposed method decrease. However, the DCF with optimal CW min significantly diminishes the decreasing rate of MAC throughput using the optimal CW min values in Fig. 2, and our proposed method outperforms both, the original DCF and the DCF with optimal CW min. Compared with the original DCF and the DCF with optimal CW min, using the optimal prioritized channel access probability in Fig. 3, our proposed method improves the MAC throughput on average by about $67.7 \%$ and by about $26.7 \%$, respectively.

\section{Conclusion}

In this paper, we proposed the prioritized channel access method to improve the MAC performance of IEEE 802.11 wireless LANs. By the proposed method, we can differentiate the nodes in backoff stage 0 and other backoff stages into different contention domains to decrease the collisions. We developed the method for obtaining the optimal prioritized channel access probability considering the MAC throughput and the service fairness between nodes.

\section{Acknowledgements}

This paper was supported by research funds from Dong-A university.

\section{REFERENCES}

[1] G. Bianchi, "IEEE 802.11-Saturation Throughput Analysis", IEEE Communications Letters, vol. 2, no. 12, pp. 318-320, 1998.

[2] IEEE Std 802.11n "Wireless LAN Medium Access Control (MAC) and Physical Layer (PHY) Specifications: Enhancements for Higher Throughput", 2009.

[3] IEEE Std 802.11ac "Wireless LAN Medium Access Control (MAC) and Physical Layer (PHY) Specifications-Amendment 4: Enhancements for Very High Throughput for Operation in Bands Below $6 \mathrm{GHz}$ ", 2013.

[4] L. Li, H. Xiaoguang, C. Ke, and H. Ketai, "The Applications of WiFi-based Wireless Sensor Network in Internet of Things and Smart Grid", Proc. IEEE Conf. on Industrial Electronics and Applications, pp. 789-793, 2011.

[5] W.-Y. Choi, and M. Chatterjee, "Cluster-Based Multipolling Sequencing Algorithm for Collecting RFID Data in Wireless LANs", FREQUENZ, vol. 69, no. 3/4, pp. 141-147, 2015.

[6] K. Medepalli and F. A. Tobagi, "On Optimization of CSMA/CA based Wireless LANs: Part I - Impact of Exponen-tial Backoff",Proc. IEEE ICC, pp. 2089-2094, 2006.

[7] X. Yang, A. O. Fapojuwo and E. E. Egbogah, "Performance Analysis and Parameter Optimization of Random Access Backoff Algorithm in LTE", Proc. IEEE VTC, pp. 1-5, 2012.

[8] B. M. Parker, J. A. Schormans and S. G. Gilmour, "Increasing Throughput in IEEE 802.11 by Optimal Selection of Backoff Parameters", IET Networks, vol. 4, no. 1, pp. 21-29, 2015.

Received 10 November 2016

Woo-Yong Choi was born in Busan, Korea 1970. He received the BS, MS and PhD degrees in industrial engineering from POSTECH (Pohang University of Science and Technology) in 1992, 1994 and 1997, respectively. From 1997 to 2001 he was a senior member of technical staff at Hyundai Electronics Industries Co., Ltd.. From 2001 to 2005 he was a senior member of technical staff at ETRI (Electronics and Telecommunications Research Institute). Since 2005 he has been with Department of Industrial \& Management Systems Engineering at Dong-A University, where he is currently a full tenured professor. Currently he is working on enhancing MAC protocols applicable for Wireless LANs. 